\title{
Effect of Anisotropic Growth and Grain Boundary Impingement on Bainite Transformation Models
}

\author{
Oskari Seppälä Aarne Pohjonen Jari Larkiola \\ Materials and Mechanical Engineering, University of Oulu, Finland, \{oskari.seppala, aarne.pohjonen, \\ jari.larkiola\} doulu.fi
}

\begin{abstract}
In the current study a previously developed cellular automata (CA) model is applied to test how the shape of the growing bainite sheaves as well as the nucleation and growth rates affect the Kolmogorov-Johnson-MehlAvrami (KJMA), Austin-Rickett (AR) and the generalized Lee-Kim (LK) model, which encompasses both the KJMA and AR models. The KJMA, AR and LK models are applied to describe the mean field evolution of the phase transformation kinetics. The shape of the growing sheaf affects the impingement phenomena, which then influences the transformation kinetics, even though the overall growth rate for different shape aspect ratios can be the same. Similarly, the different nucleation and growth rates affect the impingement. Systematic simulation studies were performed with different aspect ratios of the growing sheaves as well as different ratios of nucleation versus growth, to see the effect of these factors on the overall transformation kinetics.
\end{abstract}

Keywords: cellular automata, phase transformations, bainite, steel

\section{Introduction}

Microstructural evolution is a key factor influencing mechanical properties of steel. Evolution is based on metallurgical phenomena such as austenitization, dislocation processes, recrystallization, grain growth and phase transformations. These phenomena are controlled by process parameters like temperature, including heating and cooling rates, time, deformation and deformation rate. In addition, alloying elements of steel have a strong influence on the metallurgical phenomena. Also, the effect of the preceding process to the subsequent process stage should be included in simulations. In a complete model all these factors need be considered. However, in order to be able to construct an accurate complete model, it is necessary to understand each separate part in detail. The development cycle of new steel grades can be minimized if the final microstructure of high strength steel after TMCP (Thermo-mechanically controlled process) can be predicted. To understand how the nucleation and growth as well as the impingement of the growing bainite sheaves affect the resulting microstructure, we utilize a cellular automata (CA) model to simulate the development of bainite formation (Seppälä et al., 2018).

Although the macroscopic mean field kinetic models, such as the Kolmogorov-Johnson-Mehl-Avrami (KJMA), Austin-Rickett (A-R) or Lee-Kim (LK) models, can be fitted to experimental phase transformation data, they do not provide information on the actual microstructure. The morphology of bainite affects the kinetics because the growing bainite sheaves are obstacles for the growth of other sheaves. To simulate mesoscopic evolution of microstructure, different methods such as phase field (Militzer, 2007), CA (Militzer, 2007) and Monte Carlo (MC) (Kooi, 2006; Militzer, 2007) methods have been used. In the current study an MC-CA model has been applied, which has been developed earlier by authors (Seppälä et al., 2018) for the bainite transformation simulations, because it is capable of simulating the relevant features of the impingement phenomenon.

The CA model is used to study how the bainite sheaf shape affects the resulting morphology and the mean field kinetics described by the KJMA and A-R models. Similarly, the ratio of nucleation to growth rate on the resulting morphology and the mean field kinetics models is investigated.

\section{Theory and Calculations}

\subsection{Mean field model}

The mean field theory of bainitic transformation describing the impingement phenomena is examined by fitting the mean field model to the results obtained from the Cellular Automata (CA) model.

Perhaps the most widely used mean field model is the Kolmogorov-Johnson-Mehl-Avrami (KJMA) model, which originally introduced the extended volume concept, i.e. the volume that would be transformed if the transforming regions would not impinge with each other. If $V_{\alpha}^{e}$ is used to denote the extended volume and $V$ is the total volume, then we denote corresponding extended fraction by $\chi_{e}=V_{\alpha}^{e} / V$. In the KJMA model it is assumed that the actual transformed fraction $\chi$ is related to the extended fraction by the Equation (1)

$$
d \chi=(1-\chi)
$$

Based on continuous nucleation and growth, the extended volume fraction is calculated as $\chi_{e}=$ 
$S \int_{\tau=0}^{\tau=t} \frac{N(\tau)}{V} G(t-\tau)^{w} d \tau$, where $S$ is a shape factor related to the geometry of the growing phase, $\frac{N(\tau)}{V}$ is the nucleation rate per unit volume and $G(t-\tau)$ is the growth rate of the bainite sheaf nucleated at time $\tau$ and $w$ describes the dimension of the growing phase. For planar growth $w=2$. If nucleation and growth rates at the remaining volume stay constant, the extended volume becomes $\chi_{e}=k t^{n}$ where $k=\frac{N}{V} G$ and $n=$ $w+1$. In this case the transformed volume can be written in the widely used closed form, $\chi=1-$ $\exp \left(-k t^{n}\right)$. Even if the nucleation rate would decrease, it is still possible to fit the model reasonably well, but with a smaller value of $n$. (Christian, 1965)

As described in (Starink, 1997), a slightly different transformation model is obtained starting from Equation (2)

$$
\frac{d \chi}{d \chi_{e}}=(1-\chi)^{2}
$$

Again, assuming that the nucleation and growth rates stay constant in the remaining volume, a closed form solution is obtained: $\chi=\left(1-\frac{1}{1+k t^{n}}\right)$. This form is the Austin-Rickett (AR) model (Austin and Rickett, 1938).

According to (Starink, 1997), Lee and Kim (Lee and Kim, 1990) proposed a generalized form

$$
\frac{d \chi}{d \chi_{e}}=(1-\chi)^{1+c}
$$

which includes the parameter $\mathrm{c}$ to describe the impingement. For the value $c=0$ the equation yields the KJMA model, and for $c=1$ the equation yields the AR model.

In the current study we write the equation (3) in the form $\frac{d \chi}{d t}=(1-\chi)^{1+c} \frac{d \chi_{e}}{d t}$, where $\frac{d \chi_{e}}{d t}=n k t^{n-1}$, which we use to describe the transformation kinetics by applying difference approximation: the transformed fraction $\chi=\sum_{i} \Delta \chi$ where $\Delta \chi=\frac{d \chi}{d t} \Delta t$ is calculated for each simulation step, and $\Delta t$ is the simulation time step. The parameters $c$ and $n$ are fitted to transformed fraction calculated with the $\mathrm{CA}$ model. By changing the parameters in the CA model, their effect on the impingement parameter $c$ and the Avrami exponent $n$ can be seen.

\subsection{CA model}

Cellular Automata (CA) is a method where a simulation area is divided into equal-sized squares, cells. These cells each have a user-defined starting value, and these values can be changed by first creating neighborhood rules for the cells to follow and then applying these rules to each cell during simulation steps, which commonly depict the flow of time.

The Cellular Automata (CA) model used here is developed and first presented by authors in (Seppälä et al. 2018). Cell value depicts the phase and instance that it belongs to. In this case, the starting value of each cell is an austenitic instance, grain. During the simulation, new bainitic instances, sheaves, are initiated in austenitic grain boundaries using a probabilistic algorithm. These instances are given a growth vector and a phase instance identifier, and they gradually start to grow in the given direction with a sub-unit -like growth pattern. Figure 1 shows a simplified algorithm schema for bainitic growth. For more details on the algorithms and the equations, see (Seppälä et al., 2018).

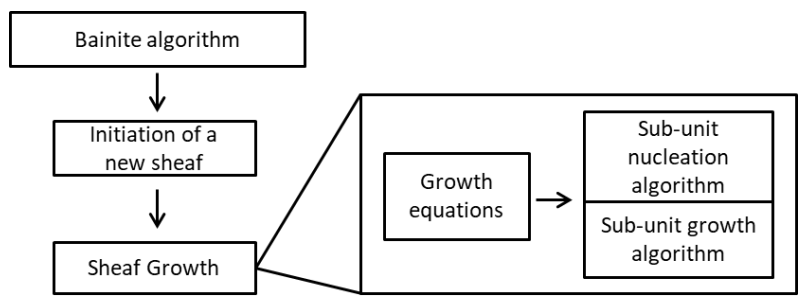

Figure 1. Cellular Automata algorithm schema for bainitic growth.

\subsection{Simulations and fitting}

The presented CA simulations are not based on experimental results. Instead the starting microstructure is generated with a self-made python script which was initially based on a static recrystallization and grain growth CA model presented in (Sieradzki and Madej, 2013). Other simulation parameters are chosen to simulate a general bainite growth simulation in constant temperature.

The parameters chosen for this study are bainite sheaf growth aspect ratio $L_{s u}$ and bainitic nucleation-growth ratio $R_{b}$. They are the main factors that can be used to influence the bainite transformation kinetics as well as the morphology of the final microstructure. The values used for fitting are $L_{s u}=4,6,8,10,12,14$, 16 and 18 and $\quad R_{b}=0.1,0.5,1.0,1.5$, 2.0, 2.5 and 3.0.

The mean field model is fitted directly into the transformation kinetics of each CA simulation case. This way the possible transformation retarding effect of $L_{s u}$ and $R_{b}$ can be linked into the physical parameters of the mean field model. The chosen fitting method is minimization of datapoint difference with equation $S_{\text {comb }}=\Sigma\left|D_{C A}-D_{M F}\right|$, where $S_{\text {comb }}$ is the sum of differences, $D_{C A}$ is a datapoint in the CA simulation and $D_{M F}$ is the mean field datapoint corresponding to the specific CA datapoint. A minimization algorithm finds the smallest possible sum of differences by changing the mean field model parameters. In this study, the truncated Newton (TNC) algorithm was used. It was implemented with Python 2.7, using the optimize.minimize function of module Scipy.

Several fitting limit combinations were tested as shown in table 1. Fitted parameters are $n$ and $c$. When the parameter name is followed by "fit", the value is fitted with the limits found on the brackets in the table. When the parameter is fixed, the name is followed by 
the fixed parameter value. From here on the fitting limit combinations will be referred to with the naming convention presented in the table.

Table 1. Mean field model fitting parameter limits.

\begin{tabular}{|c|c|c|c|c|c|}
\hline Limits & $\begin{array}{c}\mathrm{n}-3 \\
\mathrm{c}-\mathrm{fit}\end{array}$ & $\begin{array}{c}\mathrm{n} \text {-fit } \\
\mathrm{c}-\mathrm{fit}\end{array}$ & $\begin{array}{c}\mathrm{n}-2.93 \\
\mathrm{c}-\mathrm{fit}\end{array}$ & $\begin{array}{c}\mathrm{n} \text {-fit } \\
\mathrm{c}-0\end{array}$ & $\begin{array}{c}\mathrm{n} \text {-fit } \\
\mathrm{c}-1\end{array}$ \\
\hline$n$ & 3 & {$[2,3]$} & 2.93 & {$[2,3]$} & {$[2,3]$} \\
\hline$c$ & {$[-\infty, \infty]$} & {$[-\infty, \infty]$} & {$[-\infty, \infty]$} & 0 & 1 \\
\hline
\end{tabular}

\section{Results and discussion}

\subsection{CA model parameters}

Before lunging into the mean field parameters, the effect of the chosen CA model parameters $R_{b}$ and $L_{s u}$. Figure 2a) shows the effect of the parameter controlling bainite sheaf growth aspect ratio $\left(L_{s u}\right)$ on ferrite transformation kinetics. Increasing the parameter, i.e. emphasizing

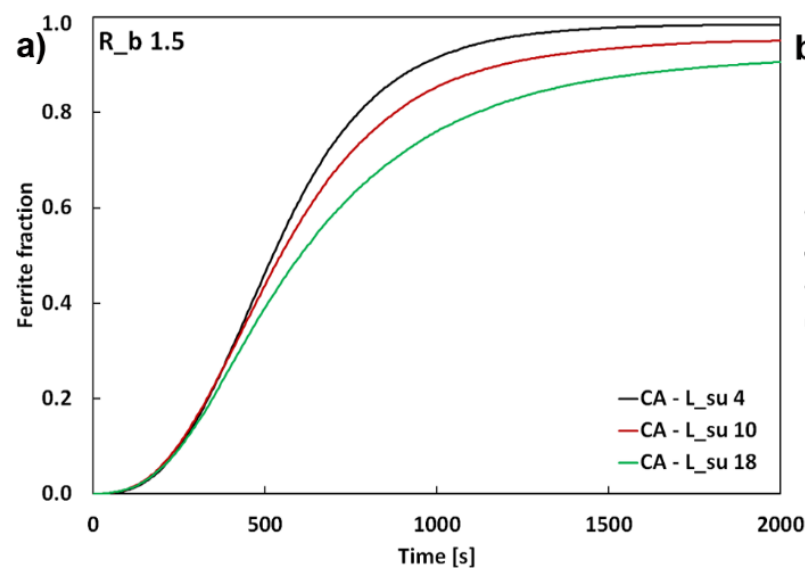

length growth, slows the later stages of transformation considerably. Figure $2 b$ ) shows the effect of the ratio of nucleation to growth rate, controlled by parameter $R_{b}$. It seems to have no clear effect on the phase transformation kinetics.

Figure 3 shows effective grain size fraction distributions with a) constant $R_{b}$ and b) constant $L_{s u}$. Included are example grain structure images illustrating the effect of varying parameters. Increasing $R_{b}$ seems to steer the distribution towards a larger amount of smaller bainite sheaves. $L_{s u}$ does not seem to have a clear effect on the distribution figure. The images show that sheaves emphasize length growth with larger values, which is to be expected.

\subsection{Mean field model parameters}

The generalized mean field model, described in the theory-section, has two parameters suitable for fitting. First is the Avrami exponent $n$, and second is the

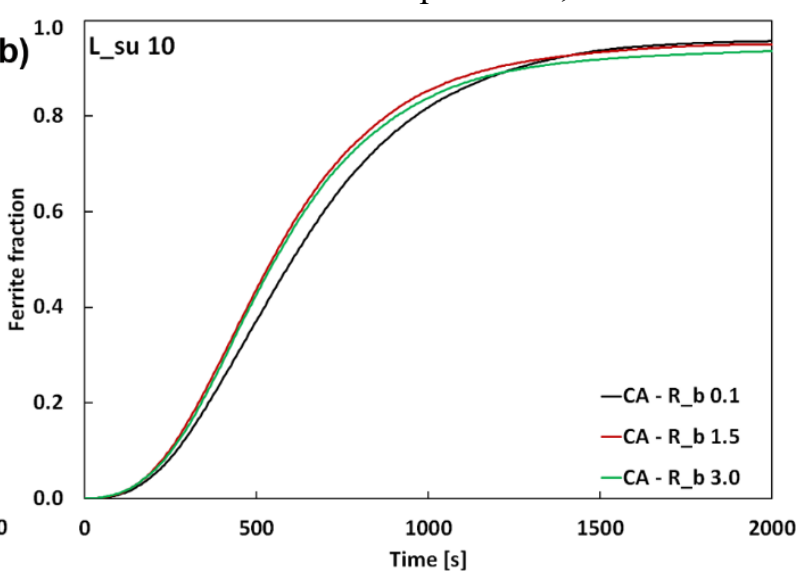

Figure 2. Bainite growth kinetics with CA model using a) constant $\boldsymbol{R}_{b}=\mathbf{1 . 5}$ with $\boldsymbol{L}_{s u}=\mathbf{4} \ldots \mathbf{1 8}$ and b) constant $\boldsymbol{L}_{s u}=\mathbf{1 0}$ with $\boldsymbol{R}_{b}=\mathbf{0 . 1} \ldots \mathbf{3 . 0}$.

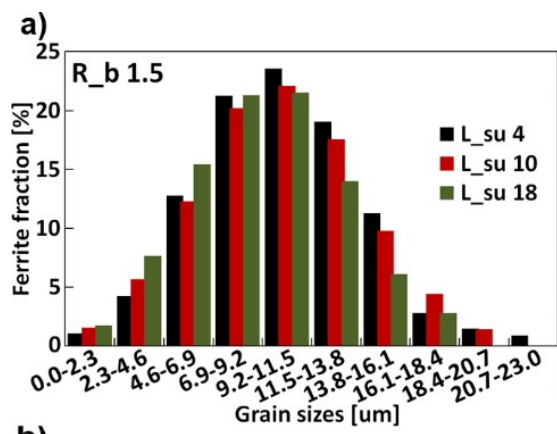

b)
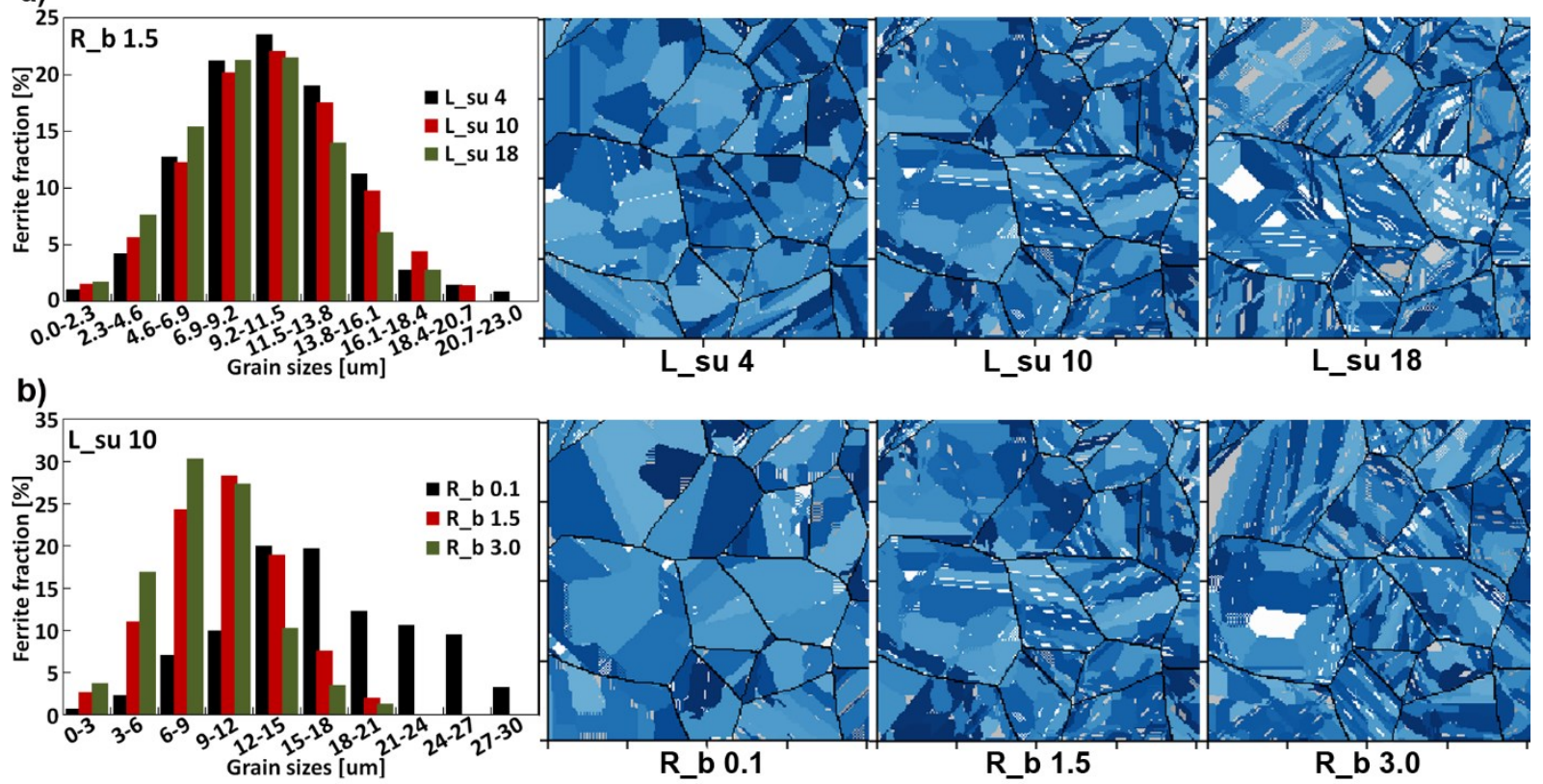

Figure 3. Effective grain size distributions and 100x100 $\mu \mathrm{m}$ sample images for bainite sheaves obtained with the CA model with a) constant $\boldsymbol{R}_{b}=\mathbf{1 . 5}$ with $\boldsymbol{L}_{\boldsymbol{s u}}=\mathbf{4} \ldots 18$ and b) constant $\boldsymbol{L}_{s u}=\mathbf{1 0}$ with $\boldsymbol{R}_{b}=\mathbf{0 . 1} \ldots \mathbf{3 . 0}$. 
impingement factor $c$. Figure 4a) includes a few selected parameter values to illustrate their effect on the transformation kinetics. Figure 4b) shows the accuracy of the chosen fitting limits with an example case where $R_{b}=1.5$ and $L_{s u}=10$. The classic KJMA equation has the impingement factor $c$ set to 0 . This clearly sets it apart from the other fitting limits, which have only minor differences.
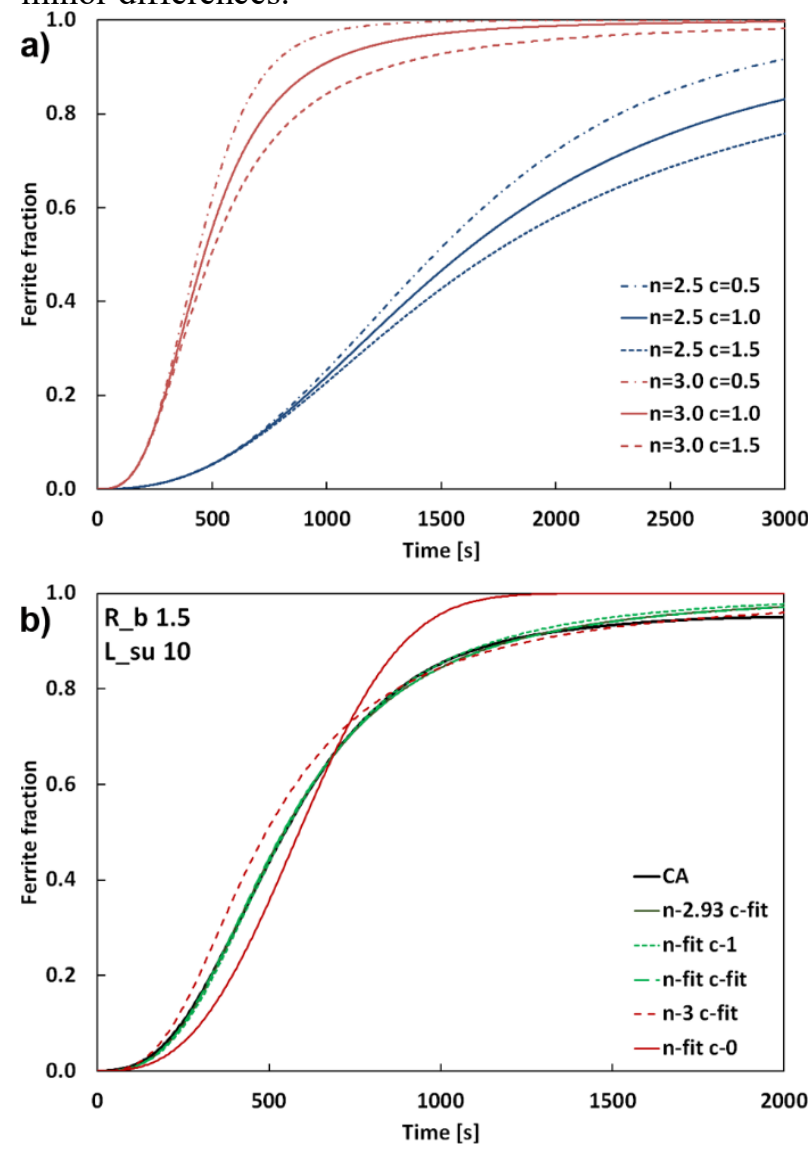

Figure 4. a) Illustrative figure of the effect of fitting parameters b) Different fitting limit examples.

A more comprehensive look on the fitting curves is provided in Figure 5. It shows the CA model phase transformation kinetics with $R_{b}=1.5$ and $L_{s u}=$ $6,10,14$ and 18. Fitting limits $n$-fit $+c$-fit were used. The correspondence between the CA results and the fitted mean field models is quite good and based on this the fitting can be called successful. The early stages of phase transformation correspond perfectly to CA data, but the later stages show minor variations. One potential problem is that the mean field model exaggerates phase transformation at the very end of the curve. The difference would be somewhat larger if the curves were to be extrapolated further. The fitting could be improved by emphasizing the end stages in the fitting procedure.

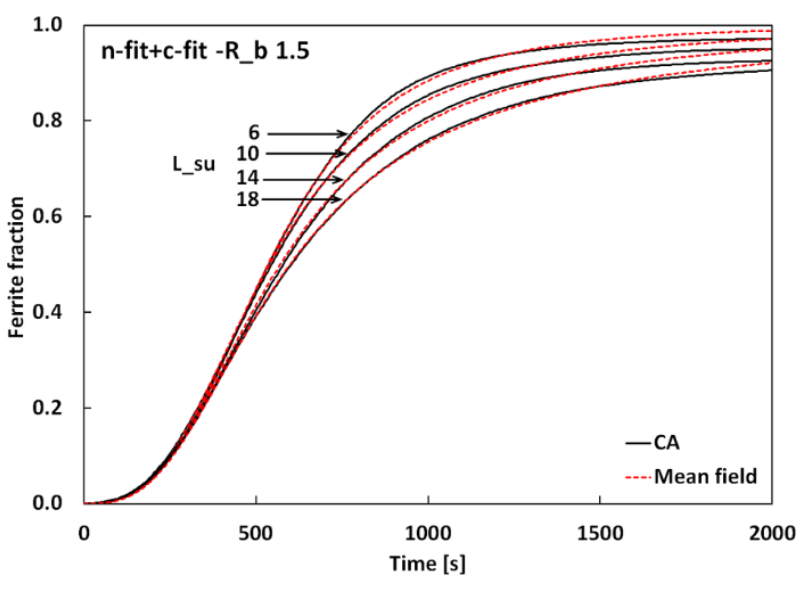

Figure 5. Mean field model fitting to CA with the n-fit+c-fit limits and $\boldsymbol{R}_{\boldsymbol{b}}=\mathbf{1 . 5}$.

The fitting parameters obtained with varying fitting limits are compared against each other by $L_{s u}$ and $R_{b}$. Figure 6 includes the fitted values for a) Avrami exponent $\mathrm{n}$ and $\mathrm{b}$ ) impingement factor $c$. Only the limits where the corresponding parameter is fitted are included in the figures. The values are grouped by $R_{b}$ as function of $L_{s u}$. For both parameters it can be seen that $R_{b}$ shows no clear effect, unlike $L_{s u}$ which shows quite a nice linear dependence for both parameters. The only notable exception is $\mathrm{n}-\mathrm{fit}+\mathrm{c}-\mathrm{fit}$, where $n$ seems to be almost a constant value. For this reason, another limit case was chosen with $n=2.93$. The chosen value is the average of $\mathrm{n}$-values from the $\mathrm{n}$-fit $+\mathrm{c}$-fit limits, excluding $R_{b}=$ 0.1 which is considered an unreliable parameter value due to the abnormal size of the sheaves produced, considering the morphology of bainite. 

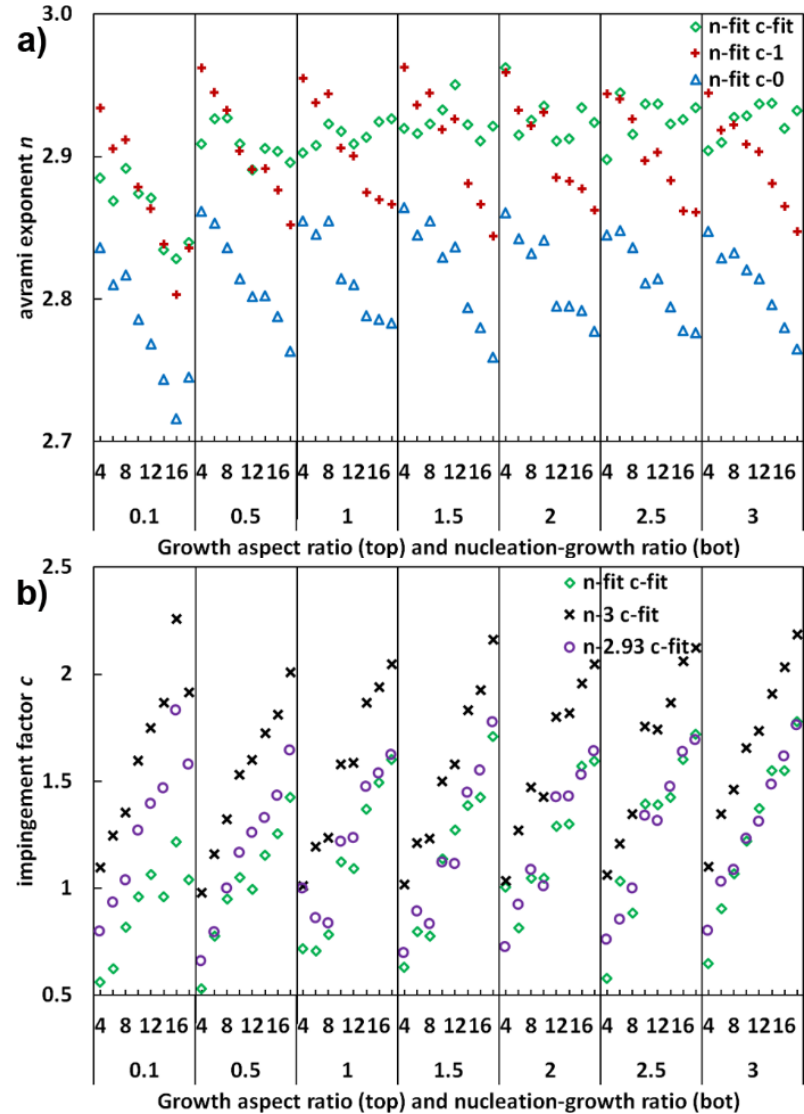

Figure 6. Fitting parameters a) $\boldsymbol{n}$ and b) $\boldsymbol{c}$, sorted by growth aspect ratio $\boldsymbol{L}_{s u}$ and nucleation-growth ratio $\boldsymbol{R}_{\boldsymbol{b}}$.

Figure 7 shows the final sums of differences for all fitting limits with a similar figure structure as in Figure 6 . The sum of differences can be used to compare the fitting limits with each other and estimate how well the fitting succeeded. Some variations are to be expected, so all the fittings can be considered successful apart from n-fit $+\mathrm{c}-0$, which is the classical KJMA equation. Not taking impingement into account shows a clear deterioration of fitting accuracy with increasing $L_{s u^{-}}$ values.

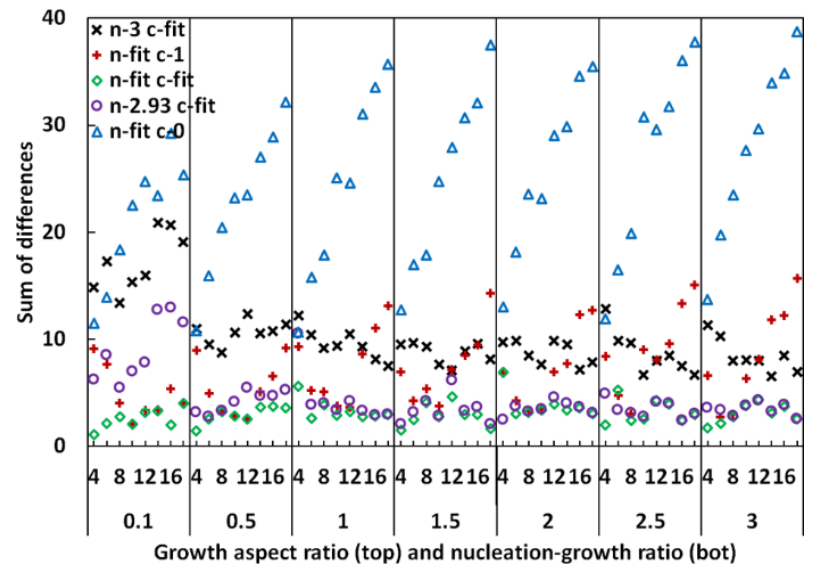

Figure 7. Comparison of the fitting accuracy of different configurations using sum of differences.

\subsection{Parameter quantification}

The results in Figure 6 are very clearly linearly dependent on $L_{s u}$. To quantify this dependence, the results were fitted linearly with the equation $y=$ $k_{y} L_{s u}+y_{0}$, where $y$ is $c$ or $n, k_{y}$ is the angular coefficient and $y_{0}$ is the intersection of the linear fit with the $y$-axis in Figure 8. Figure 8 shows fitting curves and table 2 shows linear fitting results for all $R_{b}$-values for the impingement factor $c$ with fitting limits $n-2.93+c-f i t$.
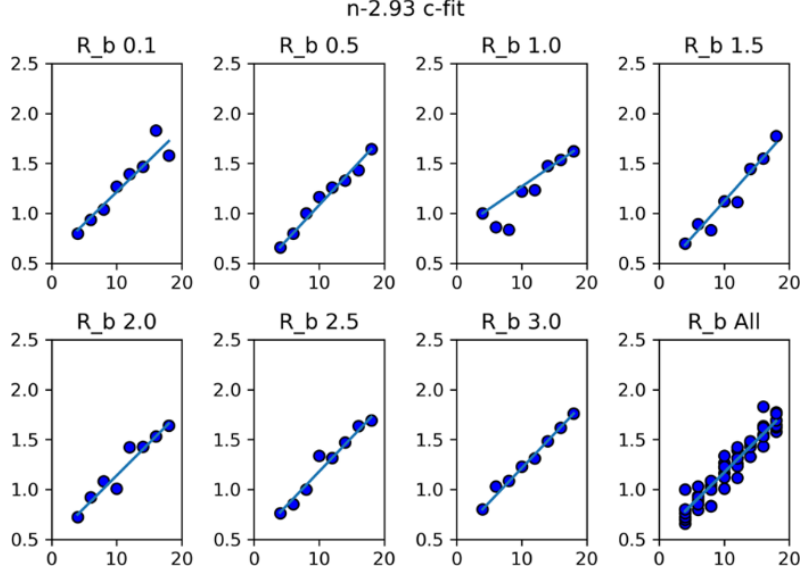

Figure 8. Linear fitting for impingement factor $c$ as function of $\boldsymbol{L}_{\boldsymbol{s}}$ with varying $\boldsymbol{R}_{\boldsymbol{b}}$ for the fitting limits n-2.93+c-fit.

Table 2. Linear fitting parameters for the impingement factor $c$ with limits n-2.93 c-fit.

\begin{tabular}{|c|c|c|c|c|}
\hline$R_{b}$ & 0.1 & 0.5 & 1 & 1.5 \\
\hline$y_{0}$ & 0.576 & 0.379 & 0.822 & 0.390 \\
\hline$k_{y}$ & 0.064 & 0.070 & 0.045 & 0.073 \\
\hline$R_{b}$ & 2 & 2.5 & 3 & All \\
\hline$y_{0}$ & 0.487 & 0.496 & 0.525 & 0.522 \\
\hline$k_{y}$ & 0.065 & 0.068 & 0.069 & 0.065 \\
\hline
\end{tabular}

The effect of $R_{b}$ on the transformation kinetics seems to be mostly random based on the results so far. To see any quantifiable difference between $R_{b}$-values, the fitting results were combined into averaged values of $y_{0}$ and $k_{y}$. These results and their sums of differences are presented for both $c$ and $n$ in Tables 3 and 4. Standard deviations are also included to estimate the accuracy of the averaging. 
Table 3. Linear fitting parameters for the impingement factor $c$ for all $R_{b}$.

\begin{tabular}{|c|c|c|c|}
\hline & $y_{0}$ & $k_{y}$ & $S_{\text {comb }}$ \\
\hline Limits & $\begin{array}{c}a v g \\
\text { (stdev) }\end{array}$ & $\begin{array}{c}a v g \\
\text { (stdev) }\end{array}$ & $\begin{array}{c}a v g \\
\text { (stdev) }\end{array}$ \\
\hline $\mathrm{n}-3$ & 0.787 & 0.074 & 0.390 \\
$\mathrm{c}-\mathrm{fit}$ & $(0.083)$ & $(0.005)$ & $(0.175)$ \\
\hline $\mathrm{n}-2.93$ & 0.525 & 0.065 & 0.448 \\
c-fit & $(0.149)$ & $(0.009)$ & $(0.199)$ \\
\hline $\mathrm{n}$-fit & 0.409 & 0.067 & 0.590 \\
c-fit & $(0.190)$ & $(0.017)$ & $(0.163)$ \\
\hline
\end{tabular}

Table 4. Linear fitting parameters for the Avrami exponent $n$ for all $R_{b}$.

\begin{tabular}{|c|c|c|c|}
\hline Limits & $\begin{array}{c}y_{0} \\
\text { (stdev) }\end{array}$ & $\begin{array}{c}k_{y} \\
\text { (stdev) }\end{array}$ & $\begin{array}{c}S_{\text {comb }} \\
\text { (stdev) }\end{array}$ \\
\hline $\mathrm{n}$-fit & 2.877 & -0.00621 & 0.055 \\
$\mathrm{c}-0$ & $(0.012)$ & $(0.00101)$ & $(0.016)$ \\
\hline $\mathrm{n}$-fit & 2.979 & -0.00720 & 0.055 \\
$\mathrm{c}-1$ & $(0.013)$ & $(0.00085)$ & $(0.017)$ \\
\hline $\mathrm{n}$-fit & 2.911 & -0.00002 & 0.074 \\
$\mathrm{c}-\mathrm{fit}$ & $(0.027)$ & $(0.00248)$ & $(0.027)$ \\
\hline
\end{tabular}

All the fitted parameters in tables 3 and 4 show only small differences in the standard deviations, with the notable exception of $\mathrm{n}$-fit $+\mathrm{c}-\mathrm{fit}$, where the standard deviation of $y_{0}$ and $k_{y}$ is larger. Also, the sums of differences $\left(S_{c o m b}\right)$ show relatively large standard deviations. These remarks combined indicate towards the idea that $R_{b}$ may have only a random effect on the time evolution of the overall bainite fraction. It is not enough to confirm this idea though, so the subject should be investigated further. However, this does not adversely affect the general applicability of the L-K model in describing the impingement phenomenon.

\section{Conclusions}

Based on the results presented here, some remarks can be made about the effects of the CA parameters chosen for investigation. Firstly, the nucleation to growth ratio, $R_{b}$, has a significant effect on the resulting effective grain size distribution but not on the phase transformation kinetics. These findings agree with the used nucleation and growth theory, and $R_{b}$ will be a useful parameter when fitting the model using experimental microstructures.

Secondly, $L_{s u}$ has no discernible effect on the effective grain size distributions, shown in Figure 3a). This is to be expected, since calculating effective grain size eliminates mathematically any anisotropy from bainite sheaves. More work needs to be done to find a method that properly quantifies the effect of growth anisotropy on simulated bainite sheaf morphology.

$L_{s u}$ has a significant effect on the phase transformation kinetics. Higher values decelerate phase transformation. This is most likely caused by growing sheaves colliding into austenite grain boundaries and other bainite sheaves, which occurs faster for sheaves with more longitudinal growth. This is the impingement effect.

The generalized mean field model has been fitted into the CA model with $c$ and $n$. The fitting results showed good agreement with the CA simulation results, and a clear correspondence between $L_{s u}$ and $c$ was found. In the currently applied theory, $c$ is the factor controlling impingement. The results of this study support the idea that $L_{s u}$ has a major impact on the impingement during the simulation. The effect of impingement was quantified for the mean field model with a linear fitting, which also showed quite good correspondence.

Within the used fitting limits some cases showed correspondence between $L_{s u}$ and $n$ as well, but when both parameters were fitted simultaneously, $n$ showed only minor variations. Based on the used theory, $n$ is expected to vary based on the diminishing amount of nucleation sites in the simulation area and the results presented here support that sentiment.

In future studies these results can be used to further investigate impingement with experimental results. The CA model will also be developed further. Multiple CA simulations with identical parameters will be run to obtain statistically more reliable results and further study the results shown in this study.

\section{Acknowledgements}

The authors gratefully acknowledge the financial support from Business Finland through ISA (Intelligent Steel Applications) project.

AP acknowledges the funding of this research activity under the auspices of Genome of Steel (Profi3) project by the Academy of Finland through Project \#311934.

The authors gratefully acknowledge the highly informative discussions and the introduction to the references (Austin and Rickett, 1938; Lee and Kim, 1990; Starink, 1997) by M Sc. Timo Manninen.

\section{References}

J. B. Austin and R. L. Rickett. Kinetics of the Decomposition of Austenite at Constant Temperature. American Institute of Mining and Metallurgical Engineers V(Technical publication n.964): 1938.

J. W. Christian. The Theory of Transformations in Metals and Alloys. Pergamon Press, 1965

J. B. Kooi. Extension of the Johnson-Mehl-AvramiKolmogorov Theory Incorporating Anisotropic Growth Studied by Monte Carlo Simulations. Phys. Rev. B 73(5): 2006. doi:10.1103/PhysRevB.73.054103 
E. S. Lee and Y. G. Kim. A Transformation Kinetic Model and Its Application to CuZnAl Shape Memory Alloys-I. Isothermal Conditions. Acta Metallurgica Et Materialia 38(9): 1669-76, 1990. doi:10.1016/0956-7151(90)90009-6

M. Militzer. Computer Simulation of Microstructure Evolution in Low Carbon Sheet Steels. ISIJ international 47(1): 1-15, 2007. doi: 10.2355/isijinternational.47.1

O. Seppälä, A. Pohjonen, A. Kaijalainen, J. Larkiola and D. Porter. Simulation of Bainite and Martensite Formation Using a Novel Cellular Automata Method. Procedia Manufacturing 15: 1856-63, 2018. doi:10.1016/j.promfg.2018.07.204

L. Sieradzki and L. Madej. A Perceptive Comparison of the Cellular Automata and Monte Carlo Techniques in Application to Static Recrystallization Modeling in Polycrystalline Materials. Computational Materials Science 67: 156-73, 2013. doi:/10.1016/j.commatsci.2012.08.047.

M. J. Starink. Kinetic Equations for Diffusion-Controlled Precipitation Reactions. Journal of Materials Science 32(15): 4061-70, 1997 doi:10.1023/A:1018649823542. 